\title{
Necessary, Sufficient and Permissive: A Single Locomotor Command Neuron Important for Intersegmental Coordination
}

\author{
Joshua G. Puhl, ${ }^{1}$ Mark A. Masino, ${ }^{1,3}$ and Karen A. Mesce ${ }^{1,2,3}$ \\ ${ }^{1}$ Graduate Program in Neuroscience, and Departments of ${ }^{2}$ Entomology and ${ }^{3}$ Neuroscience, University of Minnesota, Saint Paul, Minnesota 55108
}

In this report we posed the overarching question: What multiple contributions can a single neuron have on controlling the behavior of an animal, especially within a given context? To address this timely question, we studied the neuron R3b-1 in the medicinal leech. This bilaterally paired neuron descends from the cephalic ganglion and projects uninterrupted through the segmental ganglia comprising the nerve cord; its terminal arbors invade each hemi-ganglion. We discovered that a single R3b-1 neuron functions as a command neuron in the strictest sense, as it was both necessary and sufficient for fictive crawling behavior. Aside from these command-related properties, we determined that R3b-1 modulates the cycle period of crawl motor activity. R3b-1 has previously been shown to activate swimming behavior, but when the CNS was exposed to dopamine (DA), crawling became the exclusive locomotor pattern produced by R3b-1. DA exposure also led to bursting in R3b-1 that matched periods observed during fictive crawling, even when potential ascending inputs from crawl oscillators were removed. Although the above attributes render R3b-1 an intriguing cell, it is its ability to permit the coordination of the segmentally distributed crawl oscillators that makes this multifunctional neuron so notable. To our knowledge, this cell provides the first biological example of a single command neuron that is also vital for the intersegmental coordination of a locomotor behavior. Furthermore, our study highlights the importance of DA as an internal contextual cue that can integrate functional layers of the nervous system for adaptive behavior.

\section{Introduction}

A half century ago, Hughes and Wiersma (1960) and Wiersma and Ikeda (1964) first introduced evidence of the "command neuron," demonstrating that excitation of a single axon was capable of turning on coordinated swimmeret movements across abdominal segments in the crayfish. Wiersma and Ikeda (1964) made it clear, however, that: "the command interneurons are not part of the neural network involved in the coordinating mechanism between the different ganglia, but are superimposed on this (network)." Since these reports, the concept of the command neuron has remained embedded in the vocabulary of neuroscience, but its definitions and relevance to decision-making processes have expanded considerably (Kupfermann and Weiss, 2001; Kristan, 2008). Although Wiersma and colleagues remain correct about the swimmeret command neurons not being intersegmental coordinating interneurons (Mulloney and Smarandache-Wellmann, 2012), the possibility is examined here that a different type of command neuron might serve a coordinating role.

\footnotetext{
Received May 9, 2012; revised 0ct. 10, 2012; accepted 0ct. 13, 2012.

Author contributions: J.G.P., M.A.M., and K.A.M. designed research; J.G.P. performed research; J.G.P. analyzed data; J.G.P. and K.A.M. wrote the paper.

This work was supported by grants from the National Science Foundation (I0S-0924155 to K.A.M.) and the National Institutes of Health (individual National Research Service Award F31NS060332 to J.G.P.). We thank Gal Haspel for helpful comments concerning data analysis and the two anonymous reviewers for their constructive input. We are also especially grateful to William B. Kristan, Jr. and members of his laboratory for sharing their ideas and fruitful insights throughout the course of this work.

Correspondence should be addressed to Dr. Karen A. Mesce, Departments of Entomology and Neuroscience, University of Minnesota, 219 Hodson Hall, 1980 Folwell Avenue, St. Paul, MN 55108. E-mail: mesce001@umn.edu. DOI:10.1523/JNEUROSCI.2249-12.2012

Copyright $\odot 2012$ the authors $\quad 0270-6474 / 12 / 3217646-12 \$ 15.00 / 0$
}

A growing number of reports have provided evidence that single interneurons can influence different aspects of a behavior (Böhm and Schildberger, 1992; Hedwig, 2000) and can participate in the selection of more than one behavior depending on contextual cues (Ritzmann et al., 1980; Hooper and Moulins, 1989; Baader, 1997; Esch et al., 2002; Briggman et al., 2005; Briggman and Kristan, 2006; Berkowitz et al., 2010). Therefore, to understand what command interneurons can really do within and across contexts, and how a population-level consensus of action is formed, a timely question arises: How multifunctional can a single cell be?

In this study, we used the medicinal leech to identify the multiple roles played by a descending cephalic neuron, R3b-1 (Esch et al., 2002; Mesce et al., 2008). We tested whether R3b-1 is a bona fide classical command interneuron for crawling, fulfilling the strictest criteria of necessity and sufficiency first put forth by Kupfermann and Weiss (1978). Furthermore, we examined whether R3b-1 not only signals locomotion and initiates crawling, but permits the segmentally distributed central pattern generators (CPGs) to achieve their crawl-specific intersegmental phase relationships.

By revealing the multiple executive functions of $\mathrm{R} 3 \mathrm{~b}-1$, we aimed to underscore that even when a classical command neuron is established, as few have been, the dimensional breadth of such cells is not fully unveiled unless the contextual state of the animal is considered. Cell R3b-1, for example, does not simply command crawl production, it can activate swimming if an aquatic environmental state is presented (Esch et al., 2002).

To understand the form and dynamics of locomotion influenced by R3b-1 and the role played by the internal contextual 
state of the animal, we examined the functions of R3b-1 under the influence of dopamine (DA). We chose to study DA because our previous studies had shown that it is a potent activator of the segmental crawl CPGs (Puhl and Mesce, 2008). Here, we examined whether DA was a major contributor to the rhythmic activity of R3b1. Revisions to the original concept of the command neuron (Kupfermann and Weiss, 2001) have been based, in part, on the importance of contextual cues; they now offer us an opportunity to envision how functional layers of the nervous system are conditionally integrated for an adaptive locomotor behavior.

\section{Materials and Methods}

Animals and physiological solutions. Adult medicinal leeches (Hirudo verbana; hermaphrodites) between 1.5-3.0 g in weight were obtained from Niagara Medical Leeches and maintained at room temperature in pond water (distilled water containing dilute Instant Ocean sea salts, 1.89 g/gallon; Spectrum Brands).

Before dissection, leeches were anesthetized on ice. Dissections and all physiological recordings were performed at room temperature in normal leech saline. Normal leech saline consisted of the following (in $\mathrm{mm}$ ): $116.0 \mathrm{NaCl}, 4.0 \mathrm{KCl}, 1.8 \mathrm{CaCl}_{2}, 1.5 \mathrm{MgCl}_{2}, 10.0$ dextrose, and 10.0 Trizma preset crystals, pH 7.4 (Sigma-Aldrich) [adapted from the study by Nicholls and Baylor (1968)]. High magnesium/low calcium saline (20:1) used to block synaptic transmission contained the following (in $\mathrm{mm}$ ): $87.0 \mathrm{NaCl}, 4.0 \mathrm{KCl}, 1 \mathrm{CaCl}_{2}, 20 \mathrm{MgCl}_{2}, 10.0$ dextrose, and 10.0 Trizma preset crystals, pH 7.4 (Szczupak et al., 1998). DA-containing stock saline $(1 \mathrm{~mm})$ was prepared fresh daily and used within $5 \mathrm{~h}$. Final DA concentrations of $50-100 \mu \mathrm{M}$ for experimental use were prepared by diluting the stock with normal leech saline (Puhl and Mesce, 2008).

Leech neuroanatomy and terminology. The CNS of the medicinal leech comprises a compound cephalic ganglion (i.e., fused supraesophageal and subesophageal ganglia), 21 midbody ganglia (one per body segment designated M1-M21), and a compound tail ganglion (see schematic in Fig. 2; note here and elsewhere that the diagonal dotted lines denote ganglia not shown solely to reduce the size of the schematic representing all 21 ganglia).

The subesophageal ganglion (SEG) is divided into four neuromeres named R1-R4, each containing a dorsal and ventral layer of neuronal somata (Esch et al., 2002). The dorsal sides of R2-R4 are subdivided further into "a" and " $\mathrm{b}$ " subpackets. Cell R3b-1, a previously identified descending projection neuron that is involved in locomotion (Esch et al., 2002; Mesce et al., 2008) is thus located in the second subpacket of the third neuromere (R3b) (see Fig. 2).

For this study we monitored the activity of two uniquely identifiable motoneurons (MNs) active during crawling, the dorsal longitudinal excitor 3 (DE-3) motoneuron (MN) and the ventral lateral circular excitor (CV) MN located on the dorsal and ventral surfaces, respectively (Stuart, 1970). CV activity was monitored via intracellular recording and DE-3 by extracellular recording of the dorsal posterior (DP) nerve root, which contains the large DE-3 axon and thus most prominent extracellular spike (Kristan et al., 1974).

Experimental preparations, electrophysiological recordings, and cell identification. We removed the complete (i.e., intact) CNS from the leech body with both left and right DP nerves attached in at least 3-5 segmental ganglia. This preparation is referred to as the "whole CNS preparation" below. Fictive motor outputs were monitored by recording the activity of DE-3 extracellularly via the DP nerve. When appropriate, a Vaseline well was placed around three continuous segmental ganglia for focal application of the high magnesium/low calcium saline to block local synaptic activity; this configuration allowed the propagation of activity in fibers of passage (i.e., R3b-1) (Tschuluun et al., 2001). To induce fictive crawling, 50-100 $\mu \mathrm{M}$ DA was superfused onto the entire preparation using a peristaltic pump (Fisher Scientific). After DA perfusion and before observing coordinated fictive crawling (that propagated along the nerve cord from anterior-to-posterior), we sometimes would observe crawl-like bursting in the DP nerves that was not coordinated. During other times, DA-induced coordinated fictive crawling would not emerge until shortly after washing the DA from the preparation with normal saline (Puhl and Mesce, 2008, 2010).

Intracellular recordings of R3b-1 and CV (singly or simultaneously) were performed using an Molecular Devices AxoClamp 700B amplifier in current-clamp mode. Intracellular pipettes were filled with a solution of $2 \mathrm{M}$ potassium acetate containing $20 \mathrm{~mm}$ potassium chloride and had resistances of 30-60 $\mathrm{m} \Omega$. Electrode tips were filled with $2 \mathrm{~m}$ potassium acetate containing 5\% (w/v) Neurobiotin (Vector Laboratories). Extracellular multiunit recordings from 2-3 DP nerves were performed using suction electrodes ( $\sim 50 \mu \mathrm{m}$ tip diameter). Extracellular activity from the connectives was recorded en-passant via a suction electrode $(\sim 200 \mu \mathrm{m}$ tip diameter) so as not to damage the connective's fibers. Extracellular signals were amplified using an A-M Systems 4-channel differential ACcoupled amplifier. Both intracellular and extracellular recordings were digitized using a Digidata 16-channel data acquisition system (Molecular Devices) attached to a Microsoft Windows-compatible personal computer at 10,000 samples/s. Extracellular signals were low-pass filtered at 1 $\mathrm{kHz}$. Electrophysiological signals were visualized and plotted using pClamp (Molecular Devices), Matlab (The MathWorks), and Adobe Photoshop CS4.

In whole CNS preparations, R3b-1 was identified initially by the position and size of its soma, the existence of a correlated unit observed in the recorded connectives, as well as the emergence of either fictive swimming or crawling in response to 3-4 $\mathrm{nA}$ intracellular current injection (Esch et al., 2002). In isolated cephalic ganglion preparations (i.e., separated from the nerve cord between the SEG and M1), we first identified R3b-1 using the criteria described above, but then removed the intracellular electrode, severed the connectives between the SEG and M1, and reimpaled the neuron.

At the conclusion of each experiment the recorded R3b-1 candidate's identity was confirmed by ionophoretically filling the cell with Neurobiotin for 10-60 min to elucidate the cell's morphology. Neurobiotin was visualized by fixing and washing the tissue, then treating it with 1:50 Cy3-conjugated Streptavidin in PBS for $24-48 \mathrm{~h}$ at $4^{\circ} \mathrm{C}$. The tissue was prepared for viewing as described by Gilchrist et al. (1995). We visualized the Cy3 fluorescence using a Quantum 512SC monochrome digital camera attached to an Olympus BX61 research microscope with discscanning confocal unit (DSU) capabilities. Images were collected using MetaMorph 7 and prepared for publication in Adobe Photoshop CS4.

Dual ipsilateral hemi-connective transections and observations of overt crawling in intact leeches. We monitored crawling behaviors in intact leeches before and after dual ipsilateral hemi-connective transections above and below M2 (Fig. $3 A$, schematic). Before and during surgery leeches were placed on ice. A transverse incision $(5-10 \mathrm{~mm})$ was made over the vicinity of M2 and a ventral longitudinal slit was made to the ventral blood sinus above and below M2. Ipsilateral hemi-connective transections above and below M2 were made using fine scissors. After surgery, leeches were placed in plastic containers filled with pond water. Although we did not perform any wound-closing procedures, bleeding after surgery stopped within $30 \mathrm{~min}$. Sham surgeries were performed as described, but without severing the hemi-connectives.

We video-recorded spontaneous crawling behaviors of transected and sham controls one day before (day -1$)$ and for several weeks after surgery. Behaviors were monitored for $8 \mathrm{~min}$ in a circular plastic arena (25 $\mathrm{cm}$ diameter) containing $\sim 20 \mathrm{ml}$ of pond water to wet the bottom surface of the arena. The number of crawl cycles during each observation period was determined for each animal as was the start and end times of the elongations and contractions for five consecutive crawl cycles.

Data analysis. To calculate the temporal characteristics of R3b-1 bursting, we determined the end time of $6-10$ consecutive R3b-1 bursts (which signified the end of elongation and the beginning of the contraction phases of crawling) (Fig. 1 A) (Esch et al., 2002). We demarcated the end times of R3b-1 bursts as the time when R3b-1 spiking ceased and the membrane potential became more hyperpolarized (Fig. $1 A$, arrows). To determine the temporal characteristics of the observed fictive motor patterns, we analyzed the bursting activity of DE-3, the largest unit in our DP recordings (see above). Start times for each of 6-10 consecutive DE-3 bursts were readily discernible by visual inspection in pClamp. A group- 


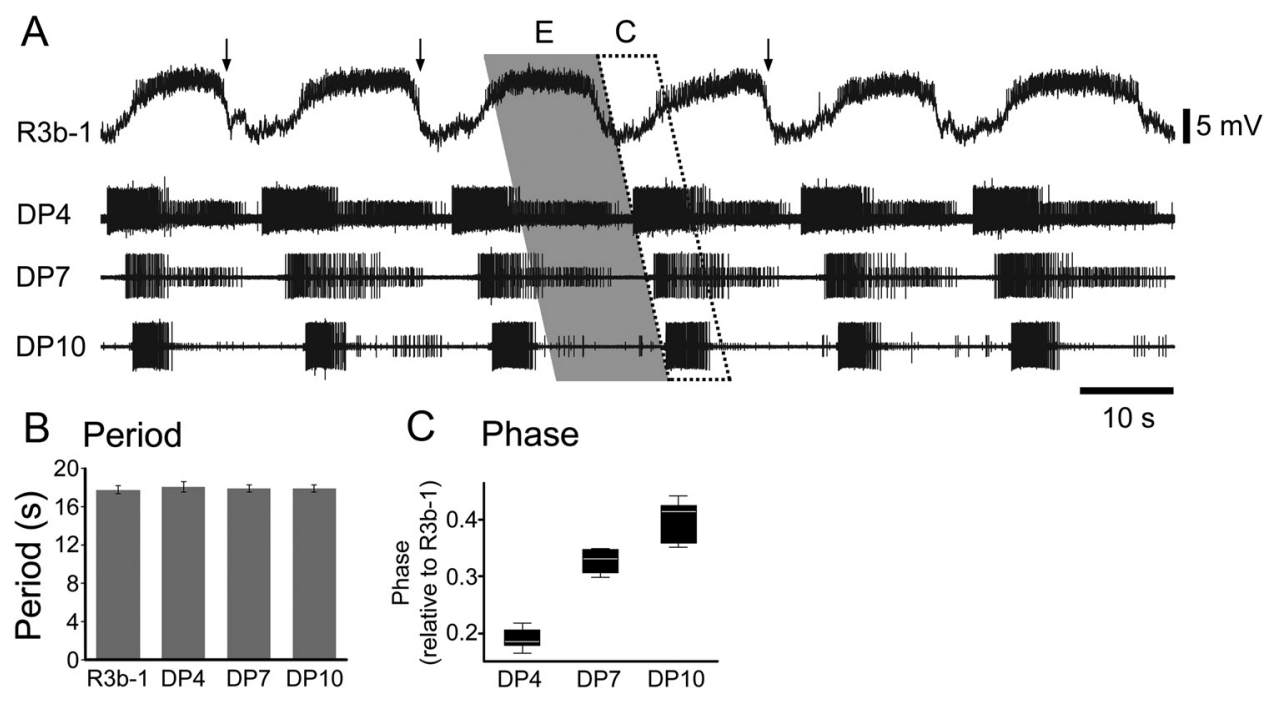

Figure 1. DA-induced spontaneous (i.e., no current injection) bursting in R3b-1 is phase-locked to fictive crawling. $A$, Shown are the DE-3 MN bursts that occur during the contraction (C) phase of fictive crawling (dotted open parallelogram, DP4-DP10); these are the largest amplitude spikes in all extracellular traces. In contrast, absence of these large units marks the elongation (E) phase (gray parallelogram, DP4-DP10). Parallelograms are extended up to the R3b-1 recording to highlight its phase locking with fictive crawling. Small units present during the elongation phase are unidentified. The MN CV, known to be active during elongation (Puhl and Mesce, 2008, 2010), is not accessible in the DP nerves. Note that the metachronal wave of DP activity propagates along the nerve cord in an anterior to posterior direction. Arrows denote both the times when the depolarized state of R3b- 1 is sharply reduced and the approximate transition points from elongation to contraction (i.e., interface of gray and open parallelograms). $\boldsymbol{B}$, The mean cycle period of R3b-1 and the mean periods of the segmental crawl bursts, all of which are indistinguishable (9 cycles/ DP recording). C, Mean phase relationships (across 9 cycles) of R3b-1 activity (at arrow) with the onset of DE-3 bursts in M4, M7 and M10.

ing of DE-3 spikes was deemed to be a burst if at least 10 spikes occurred with an interspike interval of $<1 \mathrm{~s}$, however, the vast majority of bursts contained dozens of spikes with a higher rate of DE- 3 spiking $(>2 \mathrm{~Hz})$. The period $(p)$ of bursting was calculated as the time interval between the start of consecutive DE-3 bursts. Mean periods were calculated using $6-10$ consecutive bursts. The frequency $(f)$ of fictive crawling was calculated as the reciprocal of the crawl period $(f=1 / p)$. Rhythmic fictive motor patterns with periods shorter than $2 \mathrm{~s}$ were deemed to be fictive swimming while those with periods $>5 \mathrm{~s}$ were deemed to be fictive crawling (Kristan et al., 1974; Puhl and Mesce, 2008). The shortest fictive crawling period we observed was $7 \mathrm{~s}$, thus easily partitioning fictive swimming from crawling. A minimum of three consecutive DE-3 bursts, in each segment, typically coordinated across segments was required to confirm fictive crawl activity (Eisenhart et al., 2000; Puhl and Mesce, 2010). Intersegmental delays (ISDs) between R3b-1 and DE-3 bursts or between DE-3 bursts across segments were determined by measuring the time delay of burst start times (or burst end times for R3b-1) in two segments and dividing by the number of segments that separated the two recordings. Intersegmental phase delays (phase) were calculated by dividing the ISD by the corresponding crawl period for that cycle (phase $=$ $\mathrm{ISD} / p$ ). The most anterior recording was used as a reference signal for all ISD and phase delay calculations. All mean values are presented as \pm SEM.

The firing frequency of R3b-1 was determined by counting the number of spikes observed in the intracellular recording in a $2 \mathrm{~s}$ window and dividing by $2 \mathrm{~s}$. If the intracellular trace was not visible, such as during large amplitude current injections, we determined R3b-1 spike rates in the connectives as follows. We used R3b-1 spikes in the intracellular traces as a reference to find the correct unit in the connective recording (Fig. 4B,C). A template of the connective spike was made in Clampfit based on 20-30 R3b-1 action potentials and the template matching functions of Clampfit were used to detect the number of extracellular spikes.

Two-tailed, one-way Student's $t$ tests were performed using the software package R [(R Development Core Team, 2010) http://www. R-project.org]. The confidence level used was $0.95(\alpha=0.05)$. Pairwise correlation coefficients ( $r$ values) were calculated, using Matlab, from normalized, rectified, and low-pass filtered DP recordings. For the correlation analysis, each DP trace was shifted in time so that the first DE-3 burst occurred at the beginning of the trace. This shifting facilitated the assessment of isochronicity across the recordings.

\section{Results}

Overt crawling is defined as the alternating elongation and contraction of the body with coordinated sucker attachments; segmental body movements travel exclusively as a posteriorly directed metachronal wave (Gray et al., 1938; Stern-Tomlinson et al., 1986; Puhl and Mesce, 2010). In the intact animal, the entire body becomes elongated before onset of the body contraction. Fictive crawling, in part, consists of rhythmic posteriorprogressing segmental waves of elongation (E) and contraction (C) motor activity (Fig. 1A). During the fictive contraction phase, which is typically shorter, the largest amplitude units in the DP nerve are active. These units belong to motoneuron DE-3, which excites the longitudinal muscles (Puhl and Mesce, 2008, 2010). Figure $1 A$ shows a representative example of the rhythmic oscillations observed in the membrane potential of R3b-1 during fictive crawling. These oscillations were phase-locked to the crawl motor pattern $(N=6)$. During each segmental crawl elongation (times when DE-3 bursting was absent), R3b-1 spiking was at its peak (Fig. $1 A$, gray parallelogram). Note that the circular motoneurons $(\mathrm{CV})$, known to be active during the elongation phase, were not present in these or other DP nerve recordings (Puhl and Mesce, 2008, 2010). During each segmental crawl contraction, R3b-1 spiking was reduced as it became less depolarized (Fig. $1 \mathrm{~A}$, dotted parallelogram). The mean periods of DE-3 crawl bursts across all three DP recordings and the mean period of R3b- 1 were matched (Fig. 1B; 9 cycles per DP recording). Measured over nine cycles, the phase relationships between the termination of R3b-1 bursts (Fig. $1 A$, arrows) and the DE-3 contraction-related bursts (in DP4, DP7 and DP10) were $0.19 \pm 0.01,0.33 \pm 0.01$, and $0.40 \pm 0.01$, respectively. These results indicate that: (1) the bursting in each DP nerve occurred in succession from anterior to posterior, (2) motoneuron bursting was phase-locked with the bursting of R3b-1, and (3) phase delays were stable with minimal variance (Fig. $1 C$ ). In the nearly intact body, the elongation phase of crawling is always the first one to be initiated when R3b-1 is depolarized with positive current injection (Esch et al., 2002). 

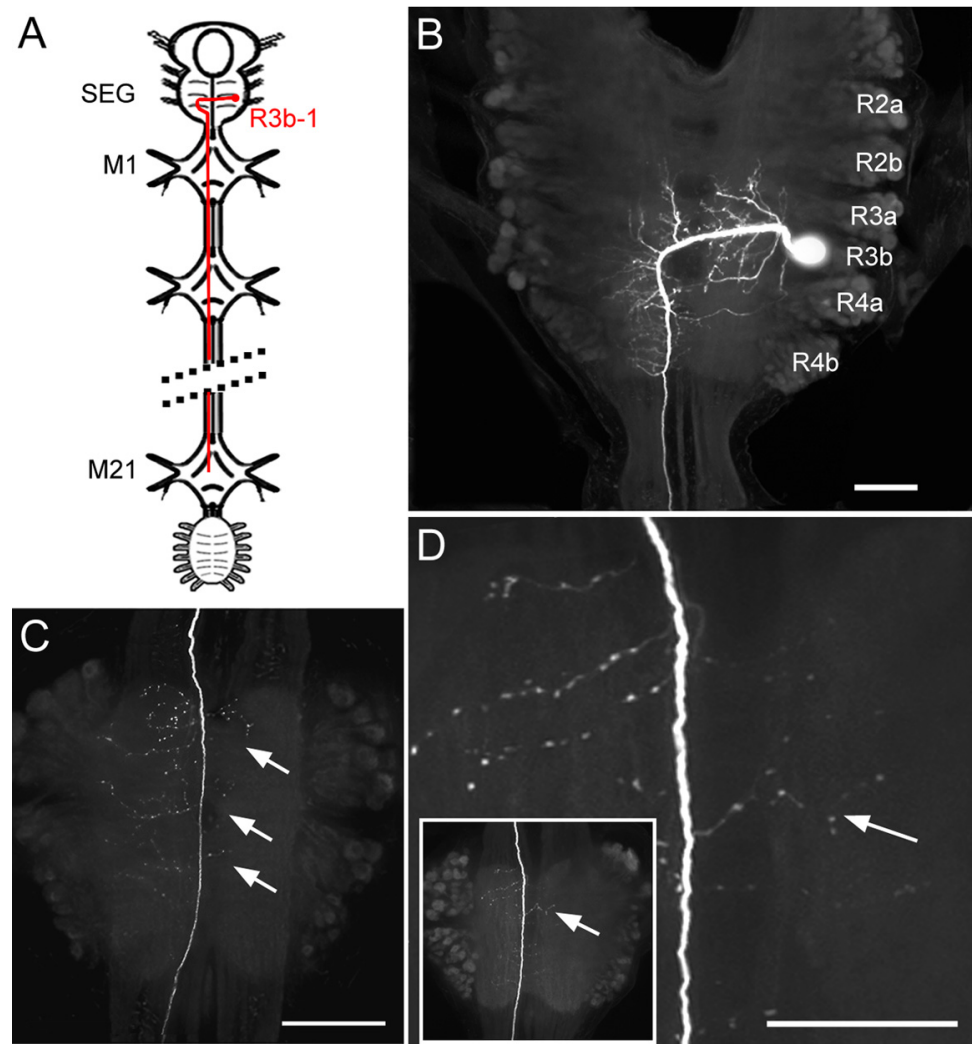

Figure 2. Morphology and projection pattern of the cephalic-originating neuron R3b-1.A, Schematic shows location of the soma and decussating primary neurite of R3b-1. The axon of this cell projects uninterrupted throughout the nerve cord; note, only one cell of the R3b-1 pair is shown. $\boldsymbol{B}$, Laser-scanning confocal micrograph of R3b-1 (dorsally situated within the SEG) that has been ionophoretically injected with Neurobiotin and visualized with Cy3 fluorescence. The neuromeres are labeled. C, Axon of R3b-1 projecting through M1. The majority of punctate arborizations ramify throughout the ipsilateral side of the ganglion; however, several arbors invade the contralateral half of the ganglion (arrows). D, Second example of the axon of R3b-1 within M1 but at a higher magnification (inset shows the entire ganglion). Arrows identify a terminal branch that crosses the midline. Scale bars: $100 \mu \mathrm{m}$.

Accounting for physical distance and putative segmental synaptic delay lines, the envelope of R3b-1 maximal activity (i.e., spikes riding on depolarized slow wave) is extrapolated to be concurrent with the elongation phase of fictive crawling initiated in the first segmental ganglion (Fig. $1 A$, gray parallelogram), substantiating the relevancy of the phase relationships between R3b-1 and the elongation phase. Such correlations thus beg the question: how dependent is crawling on R3b-1 activity? Might R3b-1 function as a crawl command neuron and be vital for intersegmental coordination? To address such questions, the following series of studies were conducted.

\section{A single cell analysis: matching the neuronal architecture of cell $\mathrm{R} 3 \mathrm{~b}$ - 1 with its role as a potential intersegmental coordinating interneuron}

R3b-1 has been described as a decussating descending projection interneuron with a soma located in the second subpacket of the third neuromere (R3b) of the subesophageal ganglion (SEG) (Esch et al., 2002); the cells are bilaterally paired. Through a series of physiological and anatomical studies, the axon of R3b-1 was shown to descend uninterrupted throughout the multiple ganglia comprising the nerve cord (Fig. 2 A, schematic; dotted lines indicate 17 ganglia omitted to limit diagram length) (Esch et al., 2002). R3b-1 has a characteristic morphology within the SEG that clearly differentiates it from other projection neurons located within the R3b packet (Esch et al., 2002; Mesce et al., 2008). Arborizations extend from the primary neurite into neighboring subpackets (R2b, R3b, R4a, and R4b) and are typically restricted to their medial half (Fig. $2 B$ ).
The morphology described above, however, does not easily explain how a single neuron has the ability to stimulate a synchronous bilateral motor response. At the level of the segmental ganglia, a potential explanation was revealed. Fine branches with punctuate regions were observed to emanate from the unilateral axon, and some of these axonal processes crossed the midline to invade the contralateral hemi-ganglion. In Figure 2, $C$ and $D$, decussating processes are shown in two different segmental ganglia. These anatomical data support the idea that a singular R3b-1 interneuron can communicate with potential crawl interneurons and motoneurons residing in both hemispheres. In addition, because the axon of R3b-1 invades the entire nerve cord (Esch et al., 2002) (see Fig. 7) all the segmental crawl circuits can be under its influence.

Although the anatomical data above support the premise that R3b-1 can communicate with crawl oscillatory networks and motoneurons throughout the nerve cord and within both hemispheres, the possibility remained that bilateral motor coordination stemmed from some type of coupling via the contralateral homolog (Fan et al., 2005) or through an intervening coupling neuron (Muller and Scott, 1980). To address this potential influence, we transected the left hemiconnective housing the R3b-1 homolog, for example below $\mathrm{M} 1$, to remove the influence of the R3b-1 located on the right (or left) side of the ganglion (soma position) (see Fig. $4 A$, micrograph). We subsequently observed overt crawl behavior using single or same-side double transections with similar results. Due to the rigorous nature of ipsilateral dual hemi-transections, we opted to report our manipulations made anterior and posterior to ganglion M2 (Fig. 3A, schematic). Before transection (day -1$)$, sham controls $(N=6)$ and experimental animals $(N=6)$ exhibited spontaneous crawling during the 8 min observation period (Fig. 3B). After these transections, we observed overt crawling behaviors (Fig. $3 B$ ) in all experimental and sham control animals. Crawl periods for transected leeches before $($ day -1$)$ and after $($ day +1$)$ transection were $7.47 \pm 2.17 \mathrm{~s}$ and $6.62 \pm 0.83 \mathrm{~s}$, respectively, and were not significantly different (Student's $t$ test; $p>0.5$ ). Elongation durations on day -1 and day +1 were $4.55 \pm 1.26 \mathrm{~s}$ and $3.75 \pm 0.67 \mathrm{~s}$, respectively, and contraction durations were $1.41 \pm 0.10 \mathrm{~s}$ and $2.11 \pm 0.36 \mathrm{~s}$ on day -1 and day +1 . The elongation and contraction durations were not significantly different after the transections compared with before (Student's $t$ test; $p>0.1$. Crawl movements from a single leech are shown in Figure $3 \mathrm{C}$, which provide additional confirmation that a single R3b-1 is sufficient for crawling.

\section{Activity of a single R3b-1 neuron is necessary and sufficient for the expression of fictive crawling that is coordinated across segments}

Having addressed the issue of whether the removal of one of the two R3b- 1 cells disrupted overt crawling, we were now in a position to test whether a single R3b-1 could be classified as classical 
command neuron, being both necessary and sufficient for crawling behavior. In addition, we aimed to determine whether R3b-1 was critical for the crawl-specific intersegmental coordination (i.e., anterior-to-posterior metachronal waves) that truly defines crawling (Puhl and Mesce, 2008, 2010). In the specific example presented here, we removed the influence of R3b-1 located in the right hemiganglion (soma) (Fig. 4A, micrograph and schematic). Both the median Faivre's nerve and contralateral hemi-connective remained unaltered, which we verified by visual inspection. Spiking in R3b-1 was also monitored in the connective (Fig. $4 B)$.

Figure $4 C$ shows the results of the above protocol in combination with 100 $\mu \mathrm{M}$ DA added to the preparation to bias a crawl state (Puhl and Mesce, 2008). At the beginning of the recording, we observed irregular bursting (burst duration and period) in each of the segmental ganglia; most importantly, crawl bursts were not phase locked to each other. In the example provided (Fig. 4C, pre-depolarization), the mean burst periods were $26.89 \pm$ $2.99 \mathrm{~s}, 28.92 \pm 1.95 \mathrm{~s}$, and $26.35 \pm 3.01 \mathrm{~s}$ for DP 8, DP 10, and DP 13, respectively, which were slightly outside the range to qualify as crawling (Puhl and Mesce, 2008, 2010). Before R3b-1 stimulation, all burst periods across the segments were not isochronous (Fig. 4D), intersegmental burst correlations were low (e.g., $r=0.34$ for DP8 and DP13), and inappropriate posterior-to-anterior phase relationships were observed (Puhl and Mesce, 2010). However, when we injected $+3 \mathrm{nA}$ of current into the functionally remaining R3b-1, coordinated fictive crawling emerged $(N=7)$. In the example shown in Figure $4 C$ (right side), intersegmental phase delays were $0.040 \pm 0.007$ between segments 8 and 10 , and $0.048 \pm 0.001$ between segments 10 and 13. The periods of DE- 3 bursting were now isochronous across all segments for every crawl cycle and the resulting shorter periods were now in the range for bona fide fictive crawling (Fig. 4E). For example, the periods during R3b-1 stimulation were $10.21 \pm$ $0.65 \mathrm{~s}$ (DP8), $10.08 \pm 0.71 \mathrm{~s}$ (DP10), and $10.24 \pm 0.69 \mathrm{~s}$ (DP13), and the burst correlation between DP8 and DP13 increased to $r=0.82$. During current injection, we relied on the extracellular unit of R3b-1 in the hemi-connective to discern spike frequency (Fig. 4B) (see Materials and Methods). During current injection, R3b-1 spiking increased dramatically (Fig. $4 C$, raster across top). Before current injection (i.e., during uncoordinated bursting), the peak frequency of R3b-1 spiking (during a $2 \mathrm{~s}$ window in the middle of the 3 bursts preceding current injection) was $8.5 \mathrm{~Hz}$ for the example shown (Fig. 4C). Three seconds after the onset of current injection, the R3b-1 spike rate was $\sim 27 \mathrm{~Hz}$, which induced coordinated fictive crawling. Increases (i.e., $>75 \%$ ) in the rate of R3b-1 spiking during the genesis of coordination was observed in all preparations analyzed $(N=4$; also treated with $\mathrm{DA})$. These results indicate that spiking activity in a single R3b-1 neuron is sufficient to command the features characteristic of fictive crawl-
B Dual ipsilateral hemi-connective transection
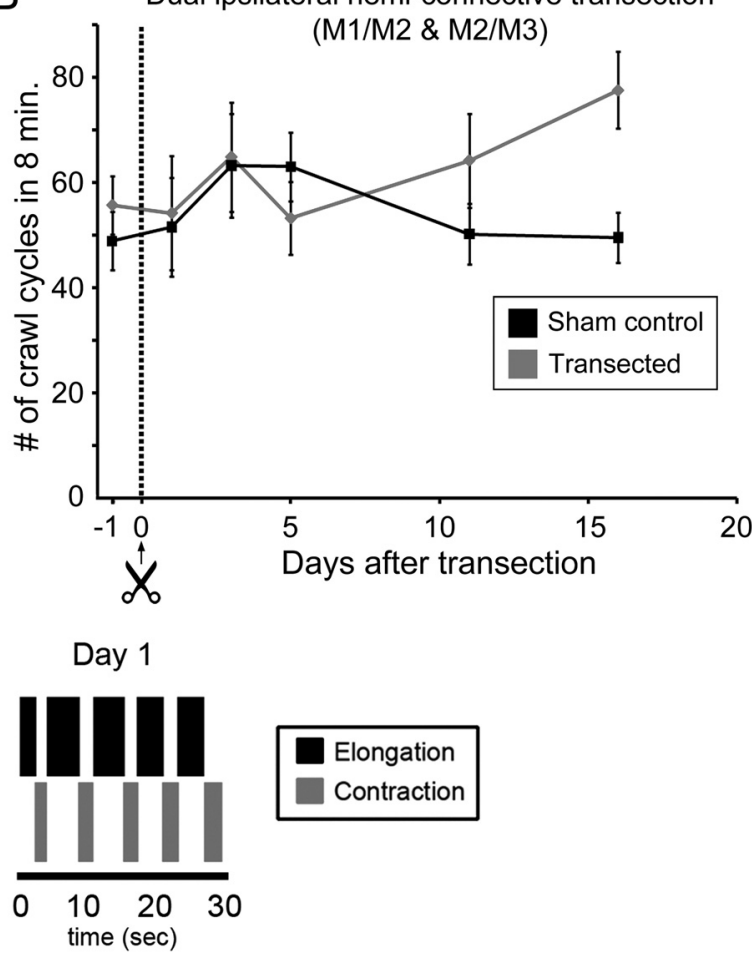

Figure 3. Transection of ipsilateral hemi-connectives anterior and posterior to a given segmental ganglion eliminate descending information from one of the pair of R3b-1 neurons but does not interrupt crawling behavior in otherwise intact leeches. $\boldsymbol{A}$, Schematic illustrates location of the dual ipsilateral hemi-connective transections. $\boldsymbol{B}$, The number of spontaneous crawl cycles 8 min was not significantly different in transected leeches $(N=6)$ and sham control leeches (i.e., received surgery but no transection) $(N=6)$. C, Crawling behaviors observed one day before and one day after dual ipsilateral hemi-connective transection were qualitatively similar, although the duration of the movements was slightly longer (data not shown).

ing within a segment and the crawl-specific coordination across segments (Puhl and Mesce, 2008, 2010).

Next, we tested whether cell R3b-1 was necessary for fictive crawling and the crawl-specific coordination across the segmental crawl oscillators. We again severed a single hemi-connective ipsilateral to the recorded R3b-1 soma to eliminate the influence of the contralateral homolog. We induced coordinated fictive crawling using $100 \mu \mathrm{M} \mathrm{DA}$ (Puhl and Mesce, 2008) and injected $-4 \mathrm{nA}$ of current to reduce R3b-1 spiking, which we verified using the connective recording (Fig. $4 F$, top raster trace). Immediately after reducing R3b-1 spiking we observed a detrimental interruption in the periodicity of DE-3 bursting in all preparations tested $(N=5)$. Most telling, in some preparations DE-3 bursting continued in some or all segments, but was accompanied by a change in the intersegmental coordination of the DE-3 bursts across all $3 \mathrm{DP}$ nerve recordings. In the example provided (Fig. $4 F$ ), some DE-3 bursting remained but the periods across segments were no longer isochronous (Fig. 4, compare $G, H$ ), which prohibited the crawl-specific intersegmental coordination of consecutive crawl cycles.

Using the strict criteria for defining fictive crawling that we and Eisenhart et al. (2000) established (see Materials and Methods), $100 \%$ of all crawl bursts in the pre-hyperpolarized condition (Fig. $4 F$ ) met these conditions. In contrast, $66 \%$ of potential crawl-like burst cycles (measured across 3 segments; 4 out of 6 cycles) failed to meet these criteria and activity could not be deemed coordinated fictive crawling. In the sample shown (Fig. $4 F$, right side) and in all other preparations $(N=5)$, when R3b-1 
A
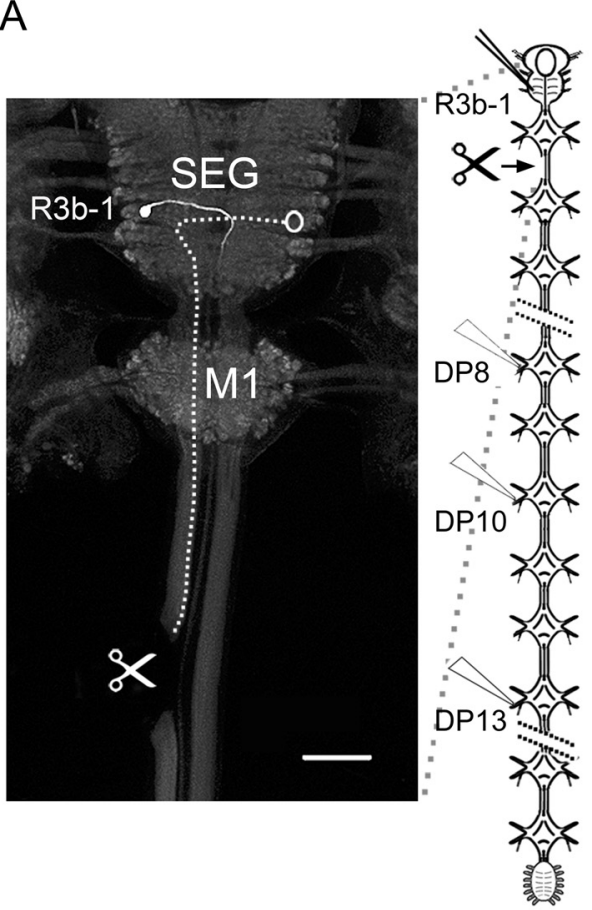

C

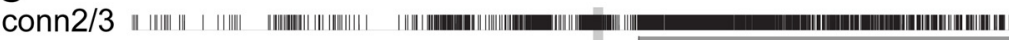

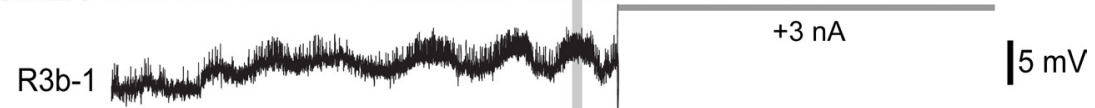

DP8

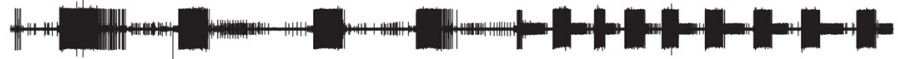

DP10

DP13

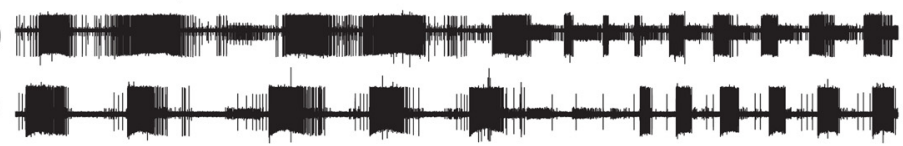

D Pre-depolarization

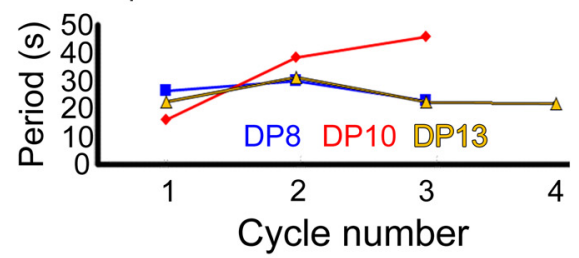

E R3b-1 Depolarized

$\overline{10 \mathrm{~s}}$

क 50

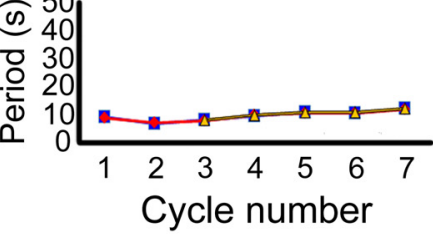

$F$

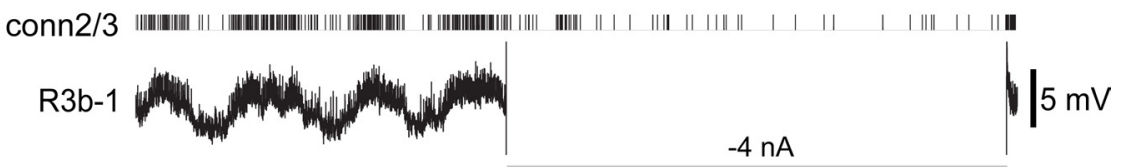

B

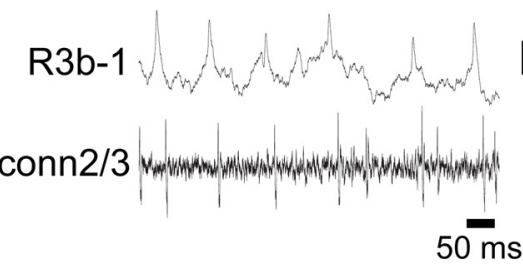

$1 \mathrm{mV}$

DP8

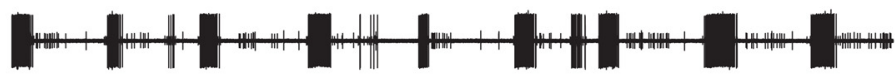

DP10

DP13

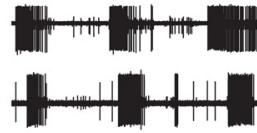

G Pre-hyperpolarization

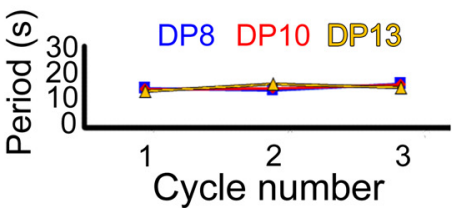

R3b-1 Hyperpolarized

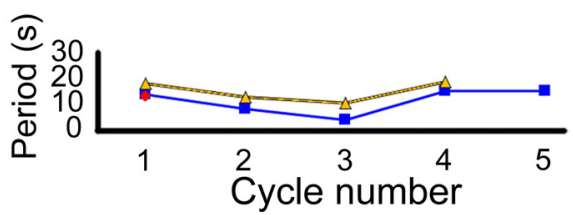

Figure 4. Spiking activity in a single R3b-1 neuron is necessary and sufficient for fictive crawling (i.e., bursting with crawl-specific intersegmental phase relationships). $A$, Confocal micrograph (left) of a transected hemi-connective between M1 and M2 (schematic right), which disrupted information flow along the axon of the contralateral R3b-1 homolog (stylized neuron). The R3b-1 recorded was filled with Neurobiotin. Note that Faivre's nerve and the contralateral hemi-connective were not damaged. Schematic (right) depicts recording sites in the cephalic ganglion and DP nerves. $\boldsymbol{B}$, Demonstration that the intracellular R3b-1 spike is identifiable in the extracellular connective recording between M2 and M3. C, Intracellular positive current injection into R3b-1 ( 3 nA; second trace) is sufficient to convert DA-induced MN bursting into fictive crawling. Top trace is a raster of R3b- 1 spike times recorded in the connectives between M2 and M3; gray vertical mark over raster and R3b-1 spikes indicates region of recording that had its time scale expanded and shown in $\boldsymbol{B}$. Fictive crawling was monitored in three DP nerves (bottom 3 traces). The largest unit in the $\mathrm{DP}$ traces belongs to the dorsal longitudinal motoneuron DE-3, which is active during the contraction phase of fictive crawling (Puhl and Mesce, 2008). Horizontal gray bar denotes $+3 \mathrm{nA}$ current injection into R3b-1 (intracellular trace is off-scale). The peak rate of R3b-1 spiking before and during current injection was $8.5 \mathrm{~Hz}$ and $27 \mathrm{~Hz}$, respectively (data not shown). $\boldsymbol{D}, \boldsymbol{E}$, The regularity and maintenance of cycle period across segments (i.e., isochronicity) is plotted before $(\boldsymbol{D})$ and after $(\boldsymbol{E})$ R3b-1 electrical stimulation. Note that R3b-1 depolarization resulted in shorter periods with all values overlapping per cycle. $\boldsymbol{F}$, Negative current injection into R3b-1 interrupted the crawl-specific intersegmental coordination that defines fictive crawling (Puhl and Mesce, 2010). Collection of cells recorded is the same as in C. Horizontal gray bar denotes time of $-4 \mathrm{nA}$ intracellular current injection (intracellular trace is off-scale). Asterisks placed below two traces highlight bursts with negative phase relationships between DP8 and DP13 (i.e., abnormal posterior-to-anterior phase relationship). Recordings in $\mathbf{C}$ and $\boldsymbol{F}$ were obtained in preparations treated with $100 \mu \mathrm{m}$ DA, which was washed off shortly before the recordings. $\mathbf{G}, \boldsymbol{H}$, Isochronicity plotted before $(\boldsymbol{G})$ and after $(\boldsymbol{H})$ R3b-1 hyperpolarization, which resulted in a disruption of isochronicity.

was hyperpolarized we never observed three consecutive cycles of crawl-specific bursting; the essential criteria defining crawling was not met. Furthermore, during R3b-1 hyperpolarization (Fig. 4F), DE-3 bursts across DP-8 and DP-13 (marked by asterisks in 2 cycles) showed phase reversals. Because leeches do not normally crawl backwards, these DE-3 phase relationships (i.e., posterior to anterior) preclude such bursting activity as constituting bona fide crawling. Looking for general DE-3 pairwise correlations ( $r$ values) across all segments recorded (Fig. $4 F$ ) (see Materials and Methods), we obtained an $r$ value of 0.14 between bursts in DP- 8 and DP- 10 when R3b-1 was hyperpolarized, which was reduced from an $r$ value of 0.64 before R3b- 1 hyperpolarization. Values across DP10 and DP13, and DP8 and DP13 were $r=0.37$ and 0.27 , respectively. Thus, although there may be some residual type of oscillator coupling in the absence of R3b-1, without the contribution of R3b-1, crawl-specific interoscillator coupling cannot be achieved as far as we have tested the system. 
A

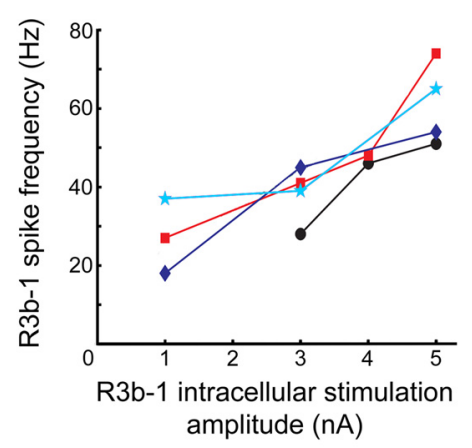

B

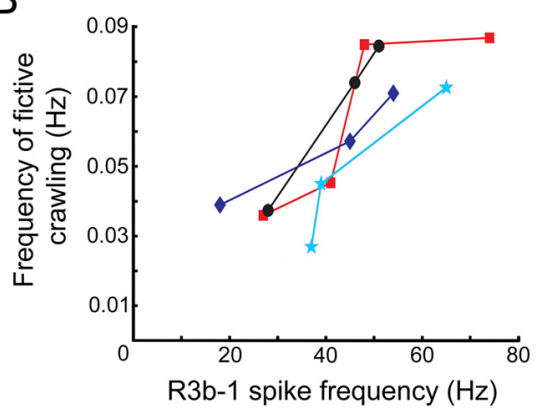

Figure 5. Scatter plots showing that the frequency of R3b-1 spiking regulates the speed of fictive crawling. $A$, Graph showing that with increased stimulus amplitude the frequency of R3b-1 spiking also increases. $\boldsymbol{B}$, Graph showing that the frequency of R3b-1 spiking is positively correlated with the frequency of fictive crawling. Data depicted in both graphs were obtained from the same preparations $(N=4)$.

\section{Mechanisms for intersegmental coordination and setting the frequency of the crawl rhythm}

Thus far the attributes of R3b-1 fulfill the strictest criteria of a classical command neuron (Kupfermann and Weiss, 1978) for, as just shown, R3b-1 is both necessary and sufficient for the activation of fictive crawling. R3b-1 was also deemed to be vital for the coordination of the intersegmental crawl oscillators. One possible way that R3b-1 might facilitate this coordination would be to ensure that the various segmental crawl oscillators share the same cycle period (i.e., be isochronous as just mentioned) (Puhl and Mesce, 2010). Figure 4, $C$ and $E$, illustrates that stimulation of a single R3b-1, indeed, has the ability to regulate the different segmental crawl periods so that they all match within each and every cycle. To extend and test the degree to which $\mathrm{R} 3 \mathrm{~b}-1$ can regulate cycle frequency, regardless of its mechanism, we drove R3b-1 with successively stronger current injections and measured whether the frequency of repeated crawl cycles changed. Again, we used 50-100 $\mu \mathrm{M}$ DA to promote crawling activity. Figure $5 \mathrm{~A}$ shows that the frequency of R3b-1 spiking increased with larger amplitudes of injected current $(N=4)$. The correlation coefficient for all data was 0.65 . Establishing that R3b-1 spike frequency and current injection were correlated, we next measured the frequency of fictive crawling in response to elevated R3b-1 spiking. Figure $5 B$ shows that the frequency of fictive crawling increased as the frequency of R3b-1 spiking was elevated $(N=4)$. The correlation coefficient for the dataset as a whole was $r=0.80$. In all four of our other preparations tested, all cycle periods were isochronous when measured across the 2-3 segments recorded, resembling the data shown in Figure 4C,E.

Intersegmental coordination of crawling requires both $\mathrm{R} 3 \mathrm{~b}-1$ spiking and local interoscillator drive

Puhl and Mesce (2010) demonstrated previously that descending cephalic signals were both necessary and sufficient for the in- tersegmental coordination of fictive crawling. Now, cell R3b-1, appears to be essential for this coordination. Puhl and Mesce (2010) also showed that local interoscillator signals exist to provide "crawl-like drive" to neighboring oscillators, extending to a minimum of two adjacent oscillators. These authors hypothesized that intersegmental coordination of the crawl oscillators might consist of a long-distance projection signal from the cephalic ganglion that functions in combination with local segmental drive. Thus, based on this idea, if intersegmental coordination were solely dependent on R3b-1 signaling, then intersegmental coordination would be preserved if the contribution from the local crawl oscillators (i.e., posterior-directed drive) were blocked. To test this idea directly, we blocked local synaptic activity across three ganglia (Fig. 6A, schematic; gray box) while allowing R3b-1, a fiber of passage, to remain relatively unaltered (see Fig. 7 for a control). Before, during, and after the synaptic blockade, we monitored fictive crawling (via DP recordings) within the blocked region and in ganglia anterior and posterior to it. Intracellular recordings of R3b-1 were maintained throughout the experiments and DA $(75-100 \mu \mathrm{M})$ was used to bias locomotion toward crawling.

Before the synaptic blockade (Fig. 6B, Pre-Block, far left), coordinated fictive crawling was observed in DP4, DP7, and DP9. R3b-1 was phase-locked to the fictive crawling rhythm in a similar manner to what was described above (Figs. 1A, 4E). Upon blocking synaptic activity in ganglia M5-M7, fictive crawling was interrupted in those ganglia within and posterior to the blocked ganglia (Fig. 6B, blocked, middle gray shaded region). Rhythmic bursting in R3b-1 was slightly altered, although it continued. Fictive crawl-like bursting was relatively uninterrupted in DP4 (anterior to the block). In the block, rhythmic activity in DP7 was disrupted within $20 \mathrm{~s}$ of the synaptic blockade and all spiking activity was abolished within several minutes. In DP9 (below the block), rhythmic activity of DE-3 continued for several cycles, appeared coordinated with bursts in DP4 for three cycles and then ceased, although other smaller DP units were still observed. We attribute the initial DE-3 coordination to the delay in the ion exchange across the glial-covered connective, which can take over a minute to complete (Puhl and Mesce, 2010), and was eventually associated with the quiescence of DP7 (Fig. 6B). Several minutes after removing the blockade, fictive crawl-like bursting continued in DP4 and coordinated bursting resumed in DP7 and DP9, with DP9 activity returning first (Fig. $6 \mathrm{~B}$, PostBlock, right). To eliminate the possibility that $\mathrm{R} 3 \mathrm{~b}-1$ spiking frequency was simply not high enough to coordinate the oscillators during the block, we stimulated R3b-1 by injecting $+3 \mathrm{nA}$ of current during a different application of the synaptic blockade in the same preparation. Figure $6 C$ shows that even during stimulation of R3b-1, no activity was observed in the blocked ganglion (DP7) and DE-3 bursting (DP9) was absent in the ganglion posterior to the block. Similar to the unstimulated condition (Fig. $6 \mathrm{~B}$ ), the ganglion anterior to the blocked ganglia (DP4) exhibited fictive crawl-like bursting of DE-3 before and during R3b-1 stimulation. Based on these data, it is evident that cell R3b-1 is necessary and sufficient for crawling, but functions in combination with local oscillator coupling so that this so-called "drive" or crawl-excitatory component is permitted to contribute to intersegmental coordination.

To validate aspects of the experiments just described, we wanted to ensure that the synaptic blockade did not interfere with the ability of R3b-1 (a fiber of passage) to make synaptic connections with potential crawl-related targets. Because specific postsynaptic targets of R3b-1 had not yet been demonstrated, we first 


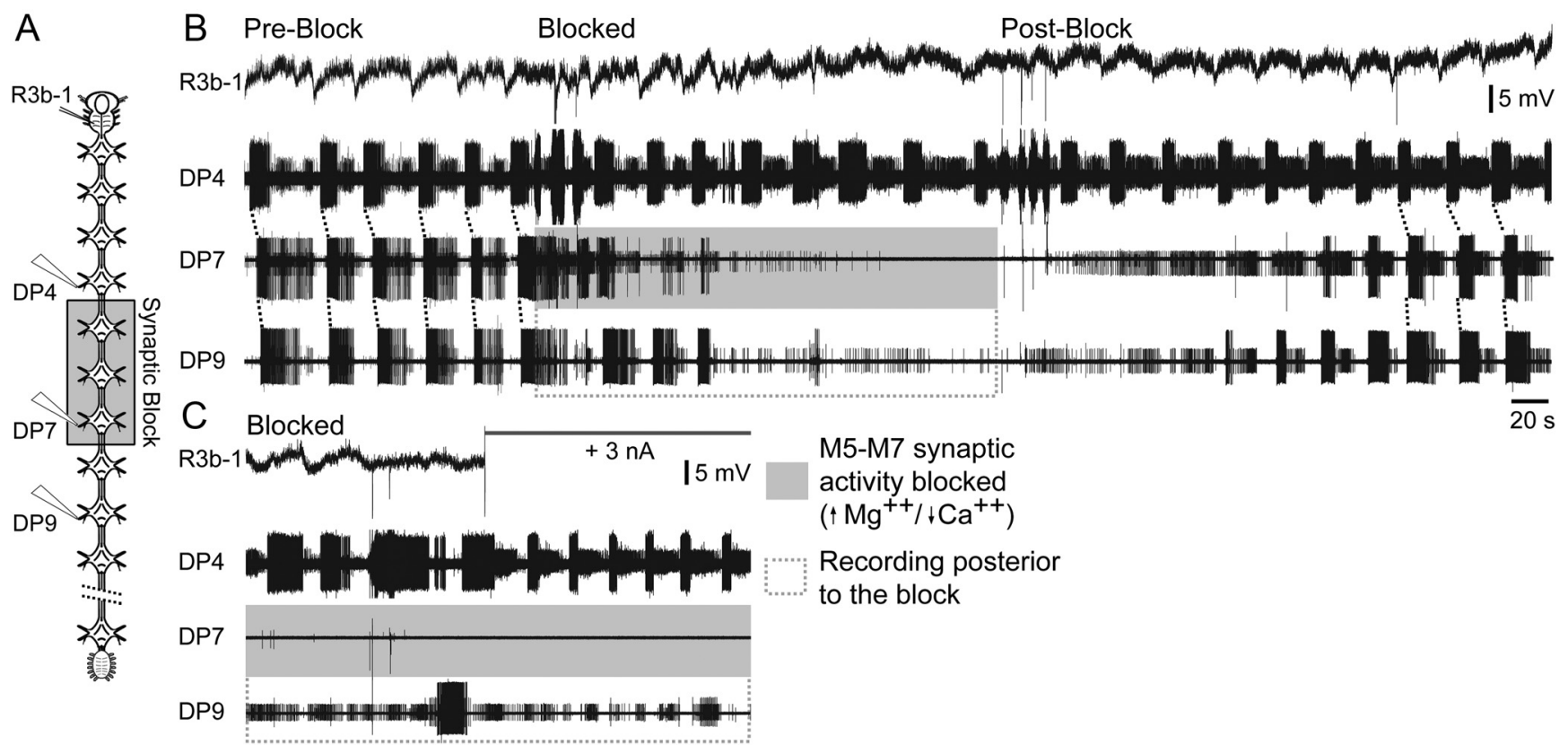

Figure 6. Fictive crawling requires parallel inputs from R3b-1 and local interoscillator circuits. $A$, Schematic showing sites of intracellular and extracellular recordings and the blockade of interoscillator signaling (gray shaded box). B, During blockade of interoscillator coupling, fictive crawling within (gray shading) and below (dotted gray box) the blocked ganglia was interrupted. Intracellular R3b-1 recording (top trace) and DP recordings from M4, M7, and M9 before (Pre-Block; left), during (Blocked; gray shaded region) and after (Post-Block; right) blockade of synaptic activity by treatment of M5-M7 with high-Mg ${ }^{2+} / \mathrm{low}_{-} \mathrm{Ca}^{2+}$ saline. Note that intersegmental coordination was present before and after blockade of synaptic activity using high Mg ${ }^{2+} / \mathrm{low} \mathrm{Ca}^{2+}$ saline (dotted black lines). C, Recording from the same preparation presented in $A$ showing the effects on fictive motor outputs of injecting $+3 \mathrm{nA}$ into R3b-1 (gray bar above trace) during synaptic blockade of M5-M7. Note: The smaller unit in DP9 recording in $\boldsymbol{C}$ is not DE-3. Horizontal calibration in $\boldsymbol{B}$ also applies to $\boldsymbol{C}$. The entire preparation was treated with $100 \mu \mathrm{m}$ DA.

needed to locate them. Based on previous models of R3b-1 connectivity (Esch et al., 2002), we tested whether R3b-1 had a synaptic connection with $\mathrm{CV}$, the $\mathrm{MN}$ known to innervate the circular muscles active during the elongation phase of crawling (Puhl and Mesce, 2008). Similar to the experiments just described (Fig. 6), we blocked local synaptic activity within and across three ganglia situated between the dual intracellular recordings of R3b-1 and MN CV $(N=3)$ (schematic, Fig. 7A, gray box). By blocking synaptic communication (Tschuluun et al., 2001) among the segmental crawl oscillators, we also reasoned that any connections observed between R3b-1 and MN CV would involve fewer interposed cells.

When R3b-1 was excited by intracellular current injection, we observed an increase in the frequency of CV spiking (Fig. $7 B$ ). Before R3b-1 current injections, the frequency of CV spiking was $2.5 \mathrm{~Hz}$. Lower amplitude current injections increased CV spiking frequency to $5 \mathrm{~Hz}$ (Fig. $7 \mathrm{~B}, 1 \mathrm{nA}$ ), whereas stronger currents increased spiking in $\mathrm{CV}$ to $8 \mathrm{~Hz}$ (also a dramatic increase in the graded potential in CV) (Fig. 7B, $3 \mathrm{nA}$ ). Stimulation of any given single R3b-1 (left or right) led to increased spiking in both the left and right CV MNs (data not shown). Figure $7 C$ shows a portion of the R3b-1 and CV recordings over a finer timescale (denoted by gray lines). We saw a consistent 1:1 R3b-1 spike to CV EPSP ratio. In the example in Figure $7 C$, the time from R3b-1 spike onset to the CV EPSP in posterior ganglion 9 was $41.1 \pm 0.29 \mathrm{~ms}$. To illustrate the relationship of R3b-1 spikes to CV EPSPs more clearly, the CV trace has been shifted to the left by $\sim 41 \mathrm{~ms}$ and correlated activity has been highlighted by gray dotted lines (Fig. 7C). Across all three preparations, we observed an EPSP in the CV recordings for $98 \%$ of R3b-1 spikes ( $N=100$ spikes per preparation). In most cases, an EPSP not observed was due to its replacement by a spike at the correct time. This study indicates that the synaptic blockade experiments did not prevent R3b-1 from signaling to its postsynaptic targets, serving as an important control for the studies in Figure 6. The R3b-1 to CV connection also illustrates that R3b-1 has the ability to influence the timing of the elongation phase of crawling directly, independent of local or interoscillator inputs.

DA locks in crawling at multiple levels of the nervous system, including cell R3b-1

In nearly intact leech preparations, electrical stimulation of the cephalic interneuron R3b-1 can elicit overt swimming or crawling depending on whether the body of the leech is suspended in fluid or touching the substrate (Esch et al., 2002). This example underscores how the contextual state of the animal sculpts adaptive behavior. In contrast, in isolated nerve cords containing the cephalic ganglia, Esch et al. (2002) observed that a given preparation did not switch between crawling or swimming and expressed only one form of fictive locomotion, typically crawling. Of 24 isolated whole CNS preparations that we tested, 19 exhibited fictive crawling and 5 produced fictive swimming in response to repeated intracellular current injections ( $2-3$ trials/animal; 3-4 nA) of R3b-1 (Fig. 8A). However, when we subsequently superfused 50-100 $\mu \mathrm{M}$ dopamine (DA) on to each of these 24 preparations, all 24 expressed fictive crawling upon repeated R3b- 1 depolarizations (2-5 trials/animal). Thus, the same 5 CNS preparations that previously showed swimming exclusively after repeated stimulation now produced solely crawling in response to identical current injection regimes (Fig. $8 \mathrm{~A}$ ). Thus the decision made by R3b-1 to locomote becomes restricted to a single form, that of crawling.

Thus far, an increase in the spiking activity of R3b-1 has been shown to activate crawling, influence intersegmental coordination, modulate the crawl cycle period, and depolarize the crawl elongation (CV) motoneurons. Might DA also cause a change in 


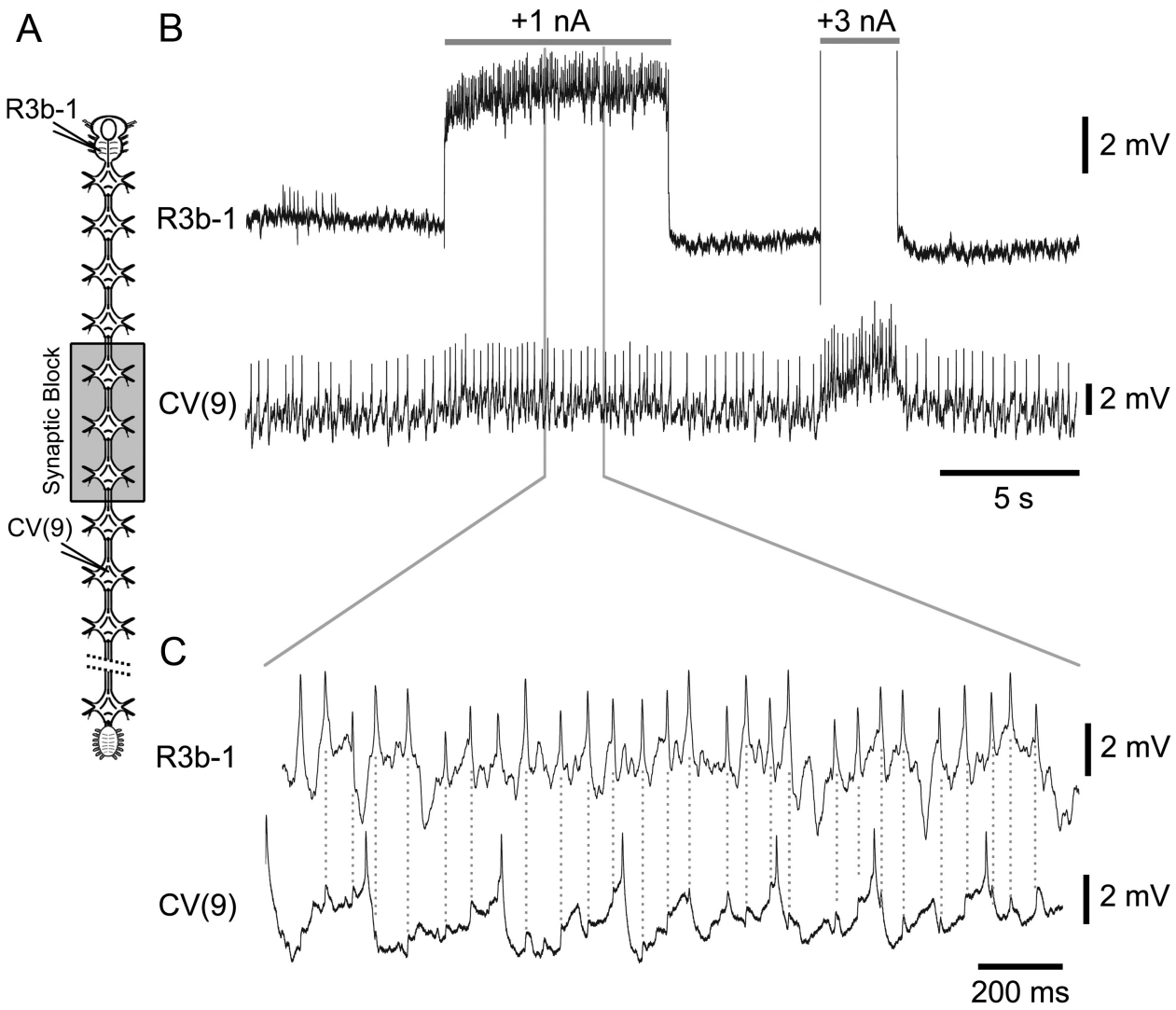

Figure 7. The crawl-related MN CV is a synaptic follower of R3b-1.A, Schematic showing electrode placement of intracellular recordings and location of synaptic blockade across three contiguous segmental ganglia (gray shaded box). This blockade disrupted interoscillator coupling (M5-M7) without perturbing information flow along the fibers of passage (i.e., R3b-1). B, Positive current injections $(+1 \mathrm{nA}$ and $+3 \mathrm{nA}$ ) led to excitation of the CV MN in M9. C, Expanded timescale of the data bracketed by vertical gray bar in $\boldsymbol{B}$. Dotted gray lines connect R3b-1 spikes to their corresponding CV EPSPs. To illustrate the relationship of R3b-1 spikes to CV EPSPs more clearly, the CV trace has been shifted to the left by $\sim 41 \mathrm{~ms}$. Under similar block conditions we observed CV EPSPs in response to R3b-1 stimulation in $100 \%$ of preparations $(N=4)$.

A

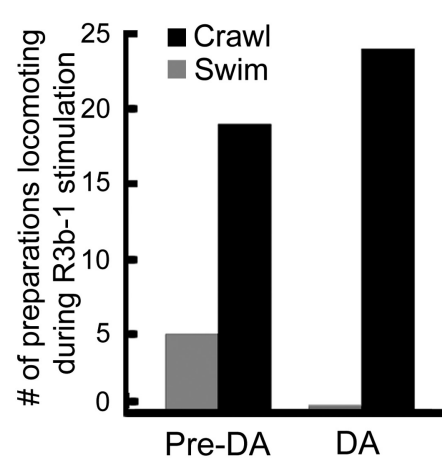

B

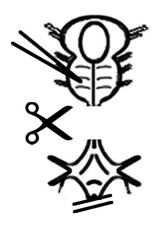

D DA treatment

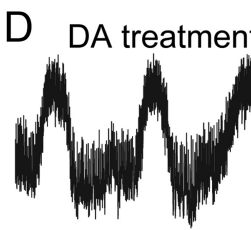

C Pre-treatment

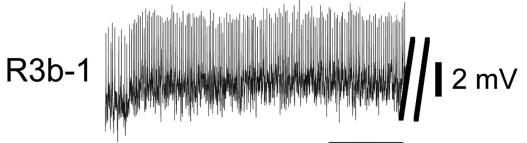

$10 \mathrm{~s}$

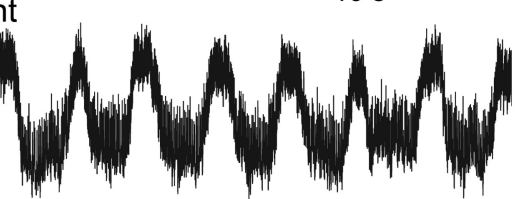

Figure 8. DA locks R3b-1 into an exclusive crawl mode and induces rhythmic oscillations in R3b-1 with a crawl-specific period. $A$, Number of whole CNS preparations $(N=24)$ in which R3b-1 was injected with positive current $(+3-4 \mathrm{nA})$ and fictive locomotion was monitored (2-3 trials/animal). Before the superfusion of 75-100 $\mu \mathrm{M} \mathrm{DA,} \mathrm{5/24} \mathrm{nerve} \mathrm{cords} \mathrm{repeatedly} \mathrm{exhibited} \mathrm{fictive}$ swimming, and no switching between fictive swimming and crawling was observed. After DA superfusion, $100 \%$ of preparations exhibited fictive crawling exclusively (3-5 trials/animal). $\boldsymbol{B}$, Schematic of the isolated cephalic ganglion preparation to remove ascending inputs and placement of electrode to record R3b-1 (see Materials and Methods for cell identification). C, R3b-1 intracellular recording before application of DA. Note that DE-3 shows tonic activity lacking any crawl-like bursting. Parallel diagonal lines at end of trace indicate continuity of the recording while DA was administered. $\boldsymbol{D}$, Intracellular recording from the same R3b-1 neuron shown in Cafter treatment with $100 \mu \mathrm{m}$ DA. Calibrations in C also apply to $\boldsymbol{D}$.

the electrical activity of R3b-1 to maximize its firing rate? If so, such an influence on R3b-1 might help to explain how DA prepares R3b-1 for its role in crawling, thus integrating executive and motor functions. Although DA has been shown to be a reliable activator of the segmental crawl oscillators (Puhl and Mesce, 2008), until now the electrical activity of R3b-1 under the influence of DA has not been reported. Because the segmental crawl CPGs are activated by DA, in the following experiments we needed to remove any potential ascending inputs from these oscillators. Thus, we recorded from R3b-1 in preparations consisting of the cephalic ganglion detached from the segmental ganglia (schematic, Fig. 8B). We observed that before a $100 \mu \mathrm{M}$ DA application, R3b-1 spiked tonically at relatively low rates in isolated cephalic preparations $(N=4)$ (Fig. $8 C)$. However, after the DA exposure, this same R3b-1 neuron exhibited rhythmic oscillations in its membrane potential with higher rates of spiking ac- 


\section{Crawl Oscillator}
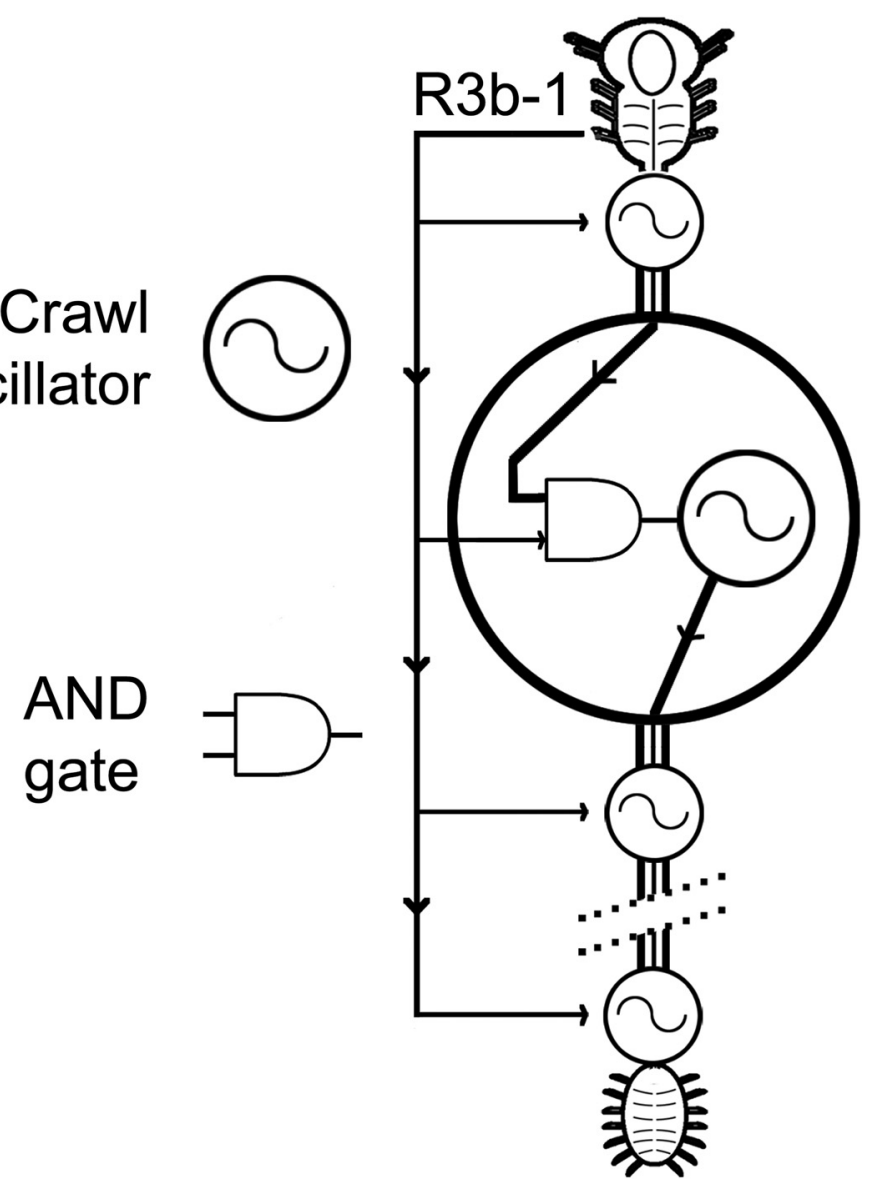

Figure 9. Schematic depicting the logical AND-gate model underlying the intersegmental coordination of crawling behavior in the medicinal leech. The inputs to the AND gate are from R3b-1 (thinner black arrows) and the anterior adjacent crawl oscillator (thicker black arrows). During crawling, R3b-1 signals are continuously present throughout the elongation phase. This R3b-1 excitation is summed with the segmental oscillator drive extending from the anterior adjacent oscillator. When the conditions of the AND gate are met, successively posterior crawl oscillators will become activated with appropriate phase relationships. Because each ganglion contains a unit burst generator for both the elongation and contraction phases of a crawl step, once the signal for crawl activation is gated, the full two-phase output will be generated (assuming a built-in delay for contraction onset).

tivity during peak depolarizations (oscillations occurred at time of application or during washout) (Fig. $8 D$ ). The average period for bursting of R3b-1 in isolated brain preparations exposed to DA was $8.1 \pm 1.3 \mathrm{~s}(N=4)$. The range was $6.1-10.6 \mathrm{~s}$, which falls within the normal range for fictive crawling of $5-25 \mathrm{~s}$ (Kristan et al., 2005; Puhl and Mesce, 2008). Because all ascending inputs from the segmental crawl oscillators were removed, the oscillatory membrane properties of R3b-1 were most likely a direct consequence of the DA application.

\section{Discussion}

\section{Overview}

In this study we established that R3b-1 is vital for the activation, maintenance, modulation, and intersegmental coordination of the segmental crawl oscillators. Specifically, R3b-1 activity is necessary for the crawl-specific coordination of DE-3 bursting propagated along the nerve cord (Fig. 4F) (Puhl and Mesce, 2010). It is also sufficient for this bursting activity and thus normal fictive crawling (Fig. 4C). When considered in isolation, the results from Figure 4 categorize R3b-1 as a command neuron for crawling using the classical definition of Kupfermann and Weiss (1978). Complementary to the role of R3b-1 in commanding crawling, we found that the rate of R3b-1 spiking influenced the iso- chronicity and cycle period observed across the segmental ganglia (Figs. $4 E$, $5 B$ ). To our knowledge, our study provides the first biological example of a single command interneuron that is essential for the intersegmental coordination of the locomotor behavior it commands.

\section{R3b-1 and a mechanism for the intersegmental coordination of crawling}

Our results confirmed and extended a prediction we made previously that a higher-order signal(s) from the cephalic ganglion is combined with local interoscillator coupling to achieve crawl-specific intersegmental coordination (Puhl and Mesce, 2010) (Fig. 9, schematic). We also found that a single R3b-1, projecting to each segmental crawl oscillator, invades both left and right hemi-ganglia (Fig. 2) and can depolarize left and right $\mathrm{CV}$ (elongation) motoneurons (Fig. 7). Our conceptual model (Fig. 9) is as follows: In each segmental ganglion, the descending inputs of R3b-1, combined with "crawl drive" from a given anterior (local) oscillator, feed into a logical AND gate to ensure the sequential activation of the next posterior crawl oscillator. For any given segmental crawl oscillator, adjacent oscillator input or that from R3b-1 alone will be subthreshold for activating the kernel for crawling in successive ganglia. Our data support this conclusion because when we blocked the interoscillator coupling among three interposed ganglia (high $\mathrm{Mg}^{2+}$, low $\mathrm{Ca}^{2+}$ ), adjacent posterior ganglia stopped bursting in a crawl mode (Fig. 6). We controlled for the potential loss of spiking activity in R3b-1 under this blockade by demonstrating that $\mathrm{MN} \mathrm{CV}$, a newly established synaptic target of R3b-1, continued to receive a 1:1 spike to EPSP ratio (Fig. 7C). When the converse experiment was conducted, whereby the input of R3b-1 was silenced with hyperpolarization (contralateral R3b-1 was functionally removed), the crawl-specific intersegmental coordination was lost (Fig. 4F). This result indicates that local oscillator-to-oscillator coupling is not sufficient to provide intersegmental coordination. A complementary experiment was conducted previously (Puhl and Mesce, 2010) in which chains of ganglia, lacking cephalic inputs, were treated with DA to activate single or multiple crawl oscillators. In all 60 cases, local oscillator coupling was never sufficient to induce intersegmental coordination, even though various segmental ganglia showed crawl-like bursting. Only when crawl networks receive dual signals can the individual crawl oscillators progressively become activated, forming a metachronal wave of posteriorly directed crawl activity (Fig. 9). Thus, our model suggests that R3b-1 not only serves as a CNS-wide crawl activation signal, but permits the concatenated posterior-directed interoscillator signaling to work. A threshold of R3b-1 spiking appears to be needed for coordination because DA-induced crawl-like activity did not become coordinated across seg- 
ments until R3b-1 spiking was sufficiently elevated through intracellular current injection (Fig. 4C). Because R3b-1 spike rates vary between individuals, crawl activation thresholds may be based on relative increases in rate (e.g., $>75 \%$ ), as we observed.

Our working model also accounts for the flexible nature of crawling. For example, crawling in intact leeches can be paused mid-cycle with other behaviors overlaid during a pause (SternTomlinson et al., 1986). If a decision is made to pause (R3b-1 is suppressed), this action would subtract one of the inputs to the crawl-oscillator's AND gates and propagation of crawl information would be terminated (Fig. 9).

Our model for intersegmental coordination offers an explanation for how relatively independent oscillators can be phase coupled by a combination of local and long-distance signals. A model with similar core features was proposed to describe a mechanism for lamprey swimming, which employs a chain of nonlinear oscillators coupled locally and by much longerdistance projecting fibers (Cohen and Kiemel, 1993). Despite differences in anatomical details, our study reflects many predictions made in the lamprey model. In contrast, swimmeret movements in the crayfish exemplify the dominance of a series of intersegmental coordinating neurons that are distributed more locally (Smarandache et al., 2009; Mulloney and SmarandacheWellmann, 2012). Reasons for such differences in modularoscillator coupling remain unanswered.

\section{The importance of context}

Our results highlight the need to examine the functional roles of neurons while considering contextual states. For example, the roles of R3b-1 as a crawl and swim activator have largely been illuminated by changing the external and internal states of the animal (Mesce and Pierce-Shimomura, 2010). Specifically, DA biases the CNS away from swimming (Crisp and Mesce, 2004) and toward crawling (Puhl and Mesce, 2008). Similar abilities of DA to modulate behavioral selection and locomotor form have been reported for Caenorhabditis elegans (Mesce and PierceShimomura, 2010; Vidal-Gadea et al., 2011).

Because R3b-1 can be considered to be a "decision-making" cell (Esch et al., 2002), the influence of DA can have widespread ramifications for behavioral selection. Here, we observed that DA can lock R3b-1 into a crawling mode (Fig. 8A), possibly preventing R3b-1 from exciting the swim circuitry, elements of which are shared with the crawl network (Briggman and Kristan, 2006). In addition, DA caused R3b-1 to exhibit rhythmic oscillations having a crawl period even when ascending information was blocked (Fig. 8D). Thus, DA can influence crawling at multiple levels of CNS organization: from the segmental crawl oscillators (Puhl and Mesce, 2008) to higher-order command cell.

The actions of DA on R3b-1 are truly elegant in light of what we have gleaned about how crawling is controlled. For example, because R3b-1 has a synaptic connection with $\mathrm{MN} \mathrm{CV}$, which elicits elongation, it makes sense that R $3 \mathrm{~b}-1$ is modulated so that its spiking is limited to the contraction phase, preventing coactivation of $\mathrm{CV}$ and its antagonist $\mathrm{MN} \mathrm{DE}-3$. In addition, maximal spike rates can be achieved when R3b-1 rhythmically reaches its depolarized plateau-like state (Figs. 1, 4, 6, 8). At these higher frequencies, R3b-1 becomes a more effective command cell (Fig. 4), can shorten the crawl periods (Fig. 5), and can theoretically contribute more input to the logical AND-gate that permits the local oscillator drive to stimulate crawl oscillators in successive segments (Fig. 9).
Because the crawl elongation and contraction phase kernels are located within each segmental "unit burst generator" (Puhl and Mesce, 2008), completion of both phases in a cycle can be initiated once the initial inputs of R3b-1 are summed, especially because R3b-1 is predominantly active during the elongation phase. Although rhythmic activity facilitates higher spike rates, elevated tonic activity is sufficient to be integrated by the logical AND gate. Other cells can produce fictive locomotion when either tonically or rhythmically active. For example, the leech swim-gating neuron, 204, can produce fictive swimming when stimulated to produce impulses tonically. Under normal conditions, however, its rhythmic activity patterns are phase locked to the swim rhythm (Weeks and Kristan, 1978).

\section{R3b-1 and the contribution of individual command-like neurons within a population}

In some systems, individual cells within a given group may contribute differentially to specific outputs or modulations of behavior (Heinrich, 2002; Kristan et al., 2005; Dubuc et al., 2008; Jordan et al., 2008; Buchanan, 2011). In the medicinal leech, specific swim command-like neurons appear more dedicated or multifunctional (Brodfuehrer and Burns, 1995; Brodfuehrer et al., 1995; Brodfuehrer et al., 2008; Mullins et al., 2011). The swim trigger neuron $\mathrm{Tr}-2$, for example, can initiate or at other times terminate swimming (Brodfuehrer and Friesen, 1986; O'Gara and Friesen, 1995). The coupling of Tr-2 with DA-containing cells may help explain why DA is an inhibitor of swimming (Crisp and Mesce, 2004).

Crickets possess command-like neurons devoted to a singular behavior, but they can impact those behaviors in multiple ways (e.g., trigger or inhibit, modulate speed, etc.) (Böhm and Schildberger, 1992; Hedwig, 2000). In the cockroach, stimulation of the command-like dorsal giant interneuron can elicit either running or flight depending on sensory cues (Ritzmann et al., 1980). Crustaceans use dedicated command-like fibers to either activate or terminate forward or backwards walking (Bowerman and Larimer, 1974) or to initiate swimmeret beating (Wiersma and Ikeda, 1964). Similar control schemes are seen in the lamprey (Buchanan and Cohen, 1982; McClellan and Grillner, 1984; Deliagina et al., 2002). Some reticulospinal neurons, for example, are dedicated to forward or backward locomotion (Zelenin, 2005), although the majority participates in both behaviors (Zelenin, 2011). Thus heterogeneity of function within cell populations and shared usage of command cells across behavioral routines appear to be a common theme emerging in the control of patterned behaviors in invertebrates and vertebrates.

As illustrated in this study, it is important to consider that as a potential member of a larger command system individual cells may have multiple functions that come and go based on contextual cues. A future challenge will be to identify the combinatorial code of a cell population for a given behavior when the functions of its individual members become continually morphed by various internal and external contextual cues.

\section{References}

Baader AP (1997) Interneuronal and motor patterns during crawling behavior of semi-intact leeches. J Exp Biol 200:1369-1381. Medline

Berkowitz A, Roberts A, Soffe SR (2010) Roles for multifunctional and specialized spinal interneurons during motor pattern generation in tadpoles, zebrafish larvae, and turtles. Front Behav Neurosci 4:36. Medline

Böhm H, Schildberger K (1992) Brain neurones involved in the control of walking in the cricket Gryllus bimaculatus. J Exp Biol 166:113-130.

Bowerman RF, Larimer JL (1974) Command fibers in the circumoesophageal connectives of crayfish II: Phasic fibers. J Exp Biol 60:119-134. 
Briggman KL, Abarbanel HD, Kristan WB Jr (2005) Optical imaging of neuronal populations during decision-making. Science 307:896-901. CrossRef Medline

Briggman KL, Kristan WB Jr (2006) Imaging dedicated and multifunctional neural circuits generating distinct behaviors. J Neurosci 26:10925-10933. CrossRef Medline

Brodfuehrer PD, Burns A (1995) Neuronal factors influencing the decision to swim in the medicinal leech. Neurobiol Learn Mem 63:192-199. CrossRef Medline

Brodfuehrer PD, Friesen WO (1986) Control of leech swimming activity by the cephalic ganglia. J Neurobiol 17:697-705. CrossRef Medline

Brodfuehrer PD, Parker HJ, Burns A, Berg M (1995) Regulation of the segmental swim-generating system by a pair of identified interneurons in the leech head ganglion. J Neurophysiol 73:983-992. Medline

Brodfuehrer PD, McCormick K, Tapyrik L, Albano AM, Graybeal C (2008) Activation of two forms of locomotion by a previously identified trigger interneuron for swimming in the medicinal leech. Invert Neurosci 8:3139. CrossRef Medline

Buchanan JT (2011) Spinal locomotor inputs to individually identified reticulospinal neurons in the lamprey. J Neurophysiol 106:2346-2357. CrossRef Medline

Buchanan JT, Cohen AH (1982) Activities of identified interneurons, motoneurons, and muscle fibers during fictive swimming in the lamprey and effects of reticulospinal and dorsal cell stimulation. J Neurophysiol 47: 948-960. Medline

Cohen AH, Kiemel T (1993) Intersegmental coordination: lessons from modeling systems of coupled non-linear oscillators. Am Zool 33:54-65.

Crisp KM, Mesce KA (2004) A cephalic projection neuron involved in locomotion is dye coupled to the dopaminergic neural network in the medicinal leech. J Exp Biol 207:4535-4542. CrossRef Medline

Deliagina TG, Zelenin PV, Orlovsky GN (2002) Encoding and decoding of reticulospinal commands. Brain Res Rev 40:166-177. CrossRef Medline

Dubuc R, Brocard F, Antri M, Fénelon K, Gariépy JF, Smetana R, Ménard A, Le Ray D, Viana Di Prisco G, Pearlstein E, Sirota MG, Derjean D, St-Pierre M, Zielinski B, Auclair F, Veilleux D (2008) Initiation of locomotion in lampreys. Brain Res Rev 57:172-182. CrossRef Medline

Eisenhart FJ, Cacciatore TW, Kristan WB Jr (2000) A central pattern generator underlies crawling in the medicinal leech. J Comp Physiol A 186:631643. CrossRef Medline

Esch T, Mesce KA, Kristan WB (2002) Evidence for sequential decision making in the medicinal leech. J Neurosci 22:11045-11054. Medline

Fan RJ, Marin-Burgin A, French KA, Otto Friesen W (2005) A dye mixture (Neurobiotin and Alexa 488) reveals extensive dye-coupling among neurons in leeches; physiology confirms the connections. J Comp Physiol A Neuroethol Sens Neural Behav Physiol 191:1157-1171. CrossRef Medline

Gilchrist LS, Klukas KA, Jellies J, Rapus J, Eckert M, Mesce KA (1995) Distribution and developmental expression of octopamine-immunoreactive neurons in the central nervous system of the leech. J Comp Neurol 353: 451-463. CrossRef Medline

Gray J, Lissman HW, Pumphrey RJ (1938) The mechanism of locomotion in the leech (Hirudo medicinalis). 15:408-430.

Hedwig B (2000) Control of cricket stridulation by a command neuron: efficacy depends on the behavioral state. J Neurophysiol 83:712-722. Medline

Heinrich R (2002) Impact of descending brain neurons on the control of stridulation, walking, and flight in orthoptera. Microsc Res Tech 56:292_ 301. CrossRef Medline

Hooper SL, Moulins M (1989) Switching of a neuron from one network to another by sensory-induced changes in membrane properties. Science 244:1587-1589. CrossRef Medline

Hughes GM, Wiersma CAG (1960) The co-ordination of swimmeret movements in the crayfish, Procambarus clarkii. J Exp Biol 37:657-670.

Jordan LM, Liu J, Hedlund PB, Akay T, Pearson KG (2008) Descending command systems for the initiation of locomotion in mammals. Brain Res Rev 57:183-191. CrossRef Medline

Kristan WB (2008) Neuronal decision-making circuits. Curr Biol 18:R928R932. CrossRef Medline

Kristan WB Jr, Stent GS, Ort CA (1974) Neuronal control of swimming in the medicinal leech I. dynamics of the swimming rhythm. J Comp Physiol 94:97-119. CrossRef
Kristan WB Jr, Calabrese RL, Friesen WO (2005) Neuronal control of leech behavior. Prog Neurobiol 76:279-327. CrossRef Medline

Kupfermann I, Weiss KR (1978) The command neuron concept. Behav Brain Sci 1:3-39. CrossRef

Kupfermann I, Weiss KR (2001) Motor program selection in simple model systems. Curr Opin Neurobiol 11:673-677. CrossRef Medline

McClellan AD, Grillner S (1984) Activation of 'fictive swimming' by electrical microstimulation of brainstem locomotor regions in an in vitro preparation of the lamprey central nervous system. Brain Res 300:357-361. CrossRef Medline

Mesce KA, Esch T, Kristan WB Jr (2008) Cellular substrates of action selection: A cluster of higher-order descending neurons shapes body posture and locomotion. J Comp Physiol A Neuroethol Sens Neural Behav Physiol 194:469-481. Medline

Mesce KA, Pierce-Shimomura JT (2010) Shared strategies for behavioral switching: understanding how locomotor patterns are turned on and off. Front Behav Neurosci 4:49. Medline

Muller KJ, Scott SA (1980) Transmission at a 'direct' electrical connexion mediated by an interneurone in the leech. J Physiol 311:565-583. Medline

Mullins OJ, Hackett JT, Friesen WO (2011) Local-distributed integration by a novel neuron ensures rapid initiation of animal locomotion. J Neurophysiol 105:130-144. CrossRef Medline

Mulloney B, Smarandache-Wellmann C (2012) Neurobiology of the crustacean swimmeret system. Prog Neurobiol 96:242-267. CrossRef Medline

Nicholls JG, Baylor DA (1968) Specific modalities and receptive fields of sensory neurons in CNS of the leech. J Neurophysiol 31:740-756. Medline

O'Gara BA, Friesen WO (1995) Termination of leech swimming activity by a previously identified swim trigger neuron. J Comp Physiol A 177:627636. Medline

Puhl JG, Mesce KA (2008) Dopamine activates the motor pattern for crawling in the medicinal leech. J Neurosci 28:4192-4200. CrossRef Medline

Puhl JG, Mesce KA (2010) Keeping it together: Mechanisms of intersegmental coordination for a flexible locomotor behavior. J Neurosci 30: 2373-2383. CrossRef Medline

R Development Core Team (2010) R: a language and environment for statistical computing. Vienna: R Foundation for Statistical Computing.

Ritzmann RE, Tobias ML, Fourtner CR (1980) Flight activity initiated via giant interneurons of the cockroach: Evidence for bifunctional trigger interneurons. Science 210:443-445. CrossRef Medline

Smarandache C, Hall WM, Mulloney B (2009) Coordination of rhythmic motor activity by gradients of synaptic strength in a neural circuit that couples modular neural oscillators. J Neurosci 29:9351-9360. CrossRef Medline

Stern-Tomlinson W, Nusbaum MP, Perez LE, Kristan WB Jr (1986) A kinematic study of crawling behavior in the leech, Hirudo medicinalis. J Comp Physiol A 158:593-603. CrossRef Medline

Stuart AE (1970) Physiological and morphological properties of motoneurones in the central nervous system of the leech. J Physiol 209:627-646. Medline

Szczupak L, Edgar J, Peralta ML, Kristan WB Jr (1998) Long-lasting depolarization of leech neurons mediated by receptors with a nicotinic binding site. J Exp Biol 201:1895-1906. Medline

Tschuluun N, Hall WM, Mulloney B (2001) Limb movements during locomotion: Tests of a model of an intersegmental coordinating circuit. J Neurosci 21:7859-7869. Medline

Vidal-Gadea A, Topper S, Young L, Crisp A, Kressin L, Elbel E, Maples T, Brauner M, Erbguth K, Axelrod A, Gottschalk A, Siegel D, PierceShimomura JT (2011) Caenorhabditis elegans selects distinct crawling and swimming gaits via dopamine and serotonin. Proc Natl Acad Sci U S A 108:17504-17509. CrossRef Medline

Weeks JC, Kristan WB Jr (1978) Initiation, maintenance and modulation of swimming in the medicinal leech by the activity of a single neuron. J Exp Biol 77:71-88.

Wiersma CA, Ikeda K (1964) Interneurons commanding swimmeret movements in the crayfish, Procambarus clarki (Girard). Comp Biochem Physiol 12:509-525. CrossRef Medline

Zelenin PV (2005) Activity of individual reticulospinal neurons during different forms of locomotion in the lamprey. Eur J Neurosci 22:2271-2282. CrossRef Medline

Zelenin PV (2011) Reticulospinal neurons controlling forward and backward swimming in the lamprey. J Neurophysiol 105:1361-1371. CrossRef Medline 